\title{
Research Paper: The Effect of Family-Centered Nature Therapy on Children With Autism Spectrum Disorder
}

\author{
Maryam Ramshini ${ }^{1}$, Saeid Hasanzadeh², Gholam Ali Afroz², Hadi Hashemi Razini ${ }^{3}$
}

1. Department of Psychology, Faculty of Literature Humanities and Social Sciences, Science and Research Branch, Islamic Azad University, Tehran, Iran.

2. Department of Psychology and Education of Exceptional Children, Faculty of Psychology and Education, University of Tehran, Tehran, Iran.

3. Department of General Psychology, Faculty of Psychology and Education, Kharazmi University, Tehran, Iran.

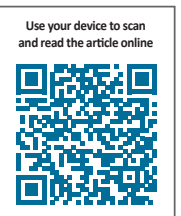

Crtation: Ramshini M, Hasanzadeh S, Afroz GhA, Hashemi Razini H. [The Effect of Family-Centered Nature Therapy on Children With Autism Spectrum Disorder (Persian)]. Archives of Rehabilitation. 2018; 19(2):150-159. http://dx.doi.org/10.32598/rj.19.2.150

doi: $:$ http://dx.doi.org/10.32598/rj.19.2.150

Received: 08 Oct 2017 Accepted: 09 Feb 2018

Keywords:

Nature therapy,

Family-centered,

Autism

\section{ABSTRACT}

Objective The treatment of autism, a long-term developmental neurological disorder, is controversial. Because of the increasing trend and the lack of a known cause in this area, the treatment is complicated; obscure development of the disorder is a fundamental issue for the parents of these children. Due to the nature of the disorder and involvement of long durations of treatment, various therapeutic methods are used. In addition to the present treatments, a cost-effective and effective treatment is nature therapy (Eco therapy). Therefore, we decided to study the effectiveness of family-centered nature therapy on children with autism spectrum disorder. Materials \& Methods A quasi-experimental (pre-test/post-test) study was conducted involving children with autism spectrum disorder (3-7 years old) who were referred to Tehran's rehabilitation and therapeutic centers. Fourteen children with autism spectrum disorder were selected by an available sampling method and randomly assigned to experimental and control groups. In each group, 7 children ( 6 boys and 1 girl) were placed. Ten therapeutic sessions were conducted in 3 months in the summer of 2017; each session was held for 3 hours (9 am to $12 \mathrm{pm}$ ) in the Nature School of Savan (located in Chitgar Forest Park) with the obligatory presence of parents (parents or at least one of them). To collect data, the Autism Treatment Evaluation Checklist (ATEC) and the Nature Therapy programs (based on the theoretical framework and relevant research findings) were used. The Autism Treatment Evaluation Checklist (ATEC) consisting of four parts, speech / language / communication, socialization, sensory / cognitive awareness, and health / physical / behavior were scrutinized three times, i.e., before the start of the session, 10 days after the last session of education and three months after the last training session. Each family was followed up by the other families. The variables studied in this study were, family-centered nature therapy (independent variable) and autism spectrum disorder syndrome (dependent variable). Data were analyzed using descriptive statistical methods (mean, standard deviation, minimum and maximum scores) and inferential methods (Leven test to assess the assumption of the equation of error variances, Kolmogorov-Smirnov test to examine the normal distribution of covariance analysis and to investigate the effect of test conditions on the dependent variable of the groups)

Results The results showed that the average score of the post-test for Autism Treatment increased in the experimental group (172.3 \pm 5.11$)$ compared with the post-test for control $(1.151 \pm 10.24)$, which was statistically significant. This showed that the nature therapy program brought an improvement in children with autism spectrum disorder. Also, the average follow-up scores (after 3 months) of the experimental group (173.91 \pm 12.02$)$ indicated the efficacy of treatment. The results of covariance analysis indicated that the calculated $F$ value $(F=21.91)$ was highly significant $(P<0.001)$, indicating the effectiveness of the experimental conditions on the dependent variable (improvement in syndrome).

Conclusion The findings of this study showed that family-centered nature therapy improved the syndrome in children with autism spectrum disorder. These children have shown remarkable progress, especially in the field of social and communication skills. Therefore, it is suggested that this type of treatment has positive, simple and accessible effects and can be used as a complementary method along with other treatments for these children.

*........

* Corresponding Author:

Saeid Hasanzadeh, PhD

Address: Department of Psychology and Education of Exceptional Children, Faculty of Psychology and Education, University of Tehran, Tehran, Iran

Tel: +98 (21) 88802214

E-Mail: shasanz@ut.ac.ir 


\title{
تأثير طبيعتدرمانى خانوادهمحور در نشانتًان كودكان طيف اتيسم
}

\author{
مريم رامشينى'، "سعيد حسنزاده'، غلامعلى افروزّ، هادى هاشمى رزينى" \\ ا - كروه روانشناسى، دانشكده ادبيات علوم انسانى و اجتماعى، واحد علوم و تحقيقات، دانشعَاه آزاد اسلامى، تهران، ايران.

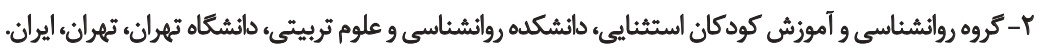

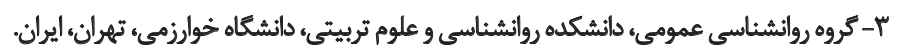

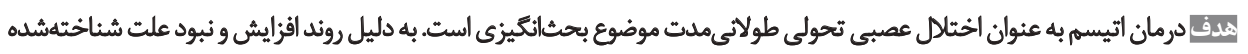

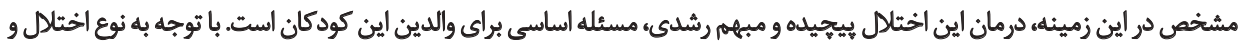

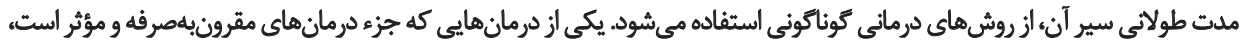

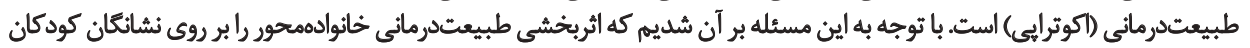

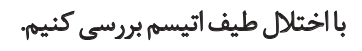

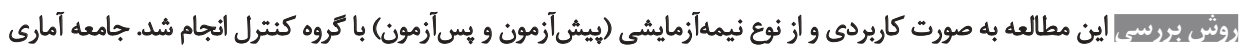

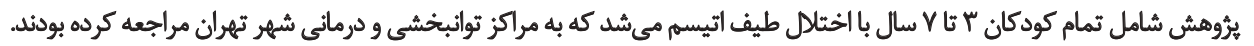

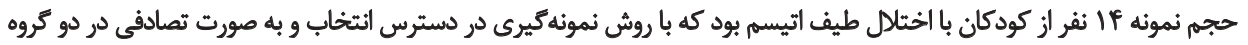

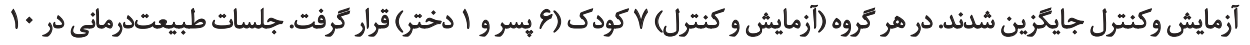

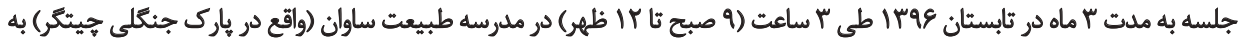

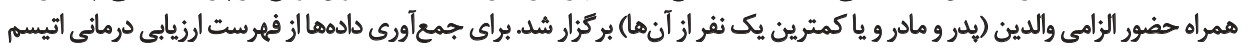

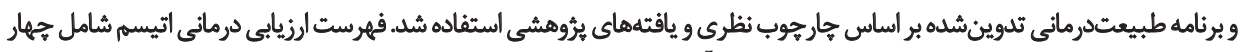

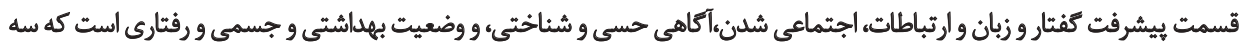

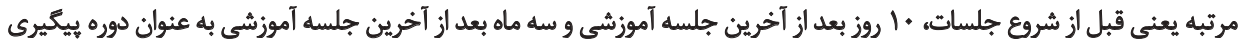

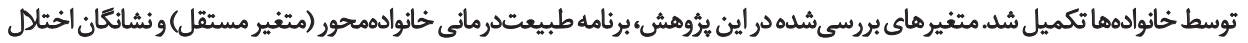

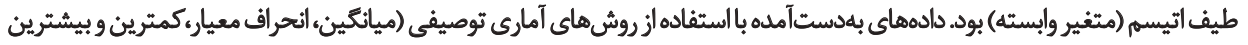

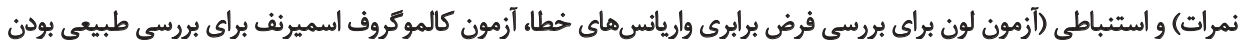

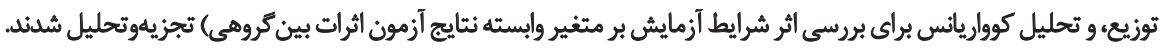

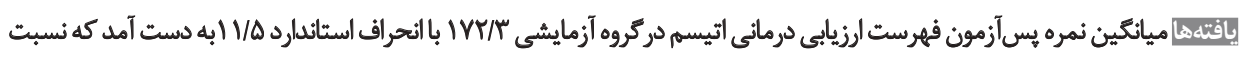

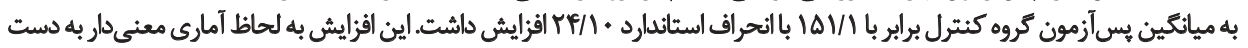

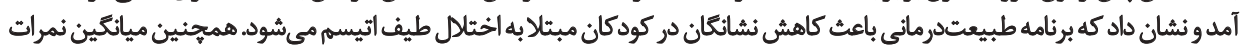

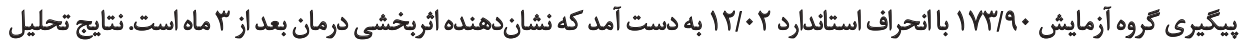

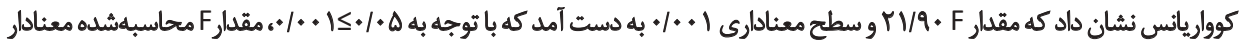

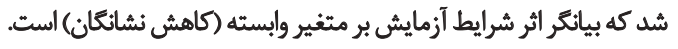

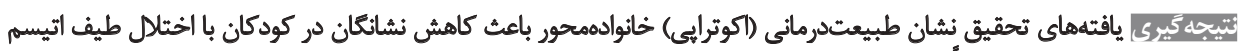

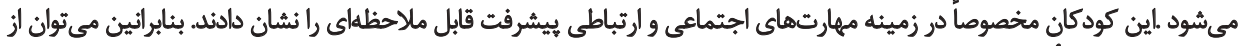

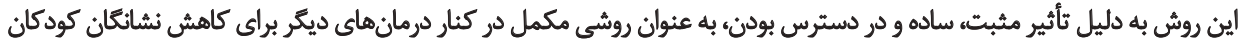
با اختيلال طيف اتيسم استفاده كرد
\end{abstract}

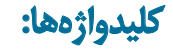

طبيعتدرمانى، اتيسهم،

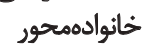




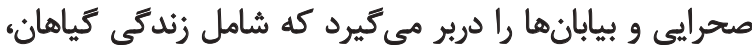
مقلمه

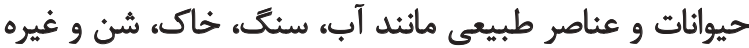

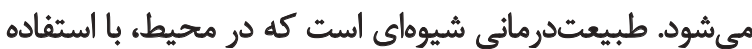

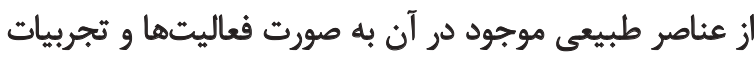

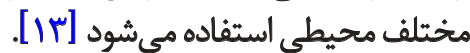
صحبث درباره طبيعتدرمانى و تأثير آن بر انسان وكاهش فشار

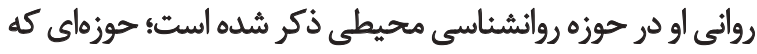

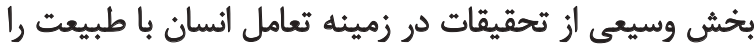

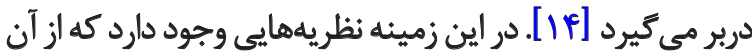

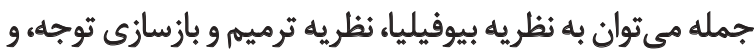
نظريه دلبستكى و حفظ محيط اشاره داشت.

نظريهييوفيليا"

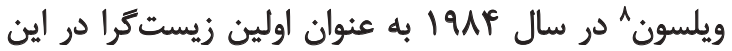

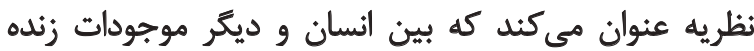

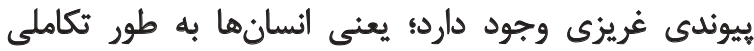

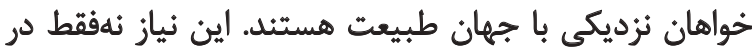

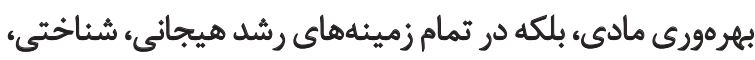
زيباشناختى و اخلاقى وجود دارد.

$$
\text { نظريه ترميمر وبازٔسازى توجه }
$$

اولين باركايلان و كايلان در سال 1919 إبا تمركز روى فرايند

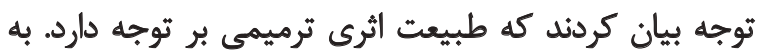

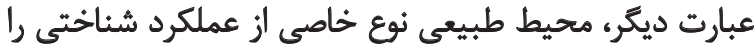

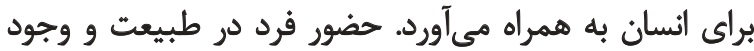

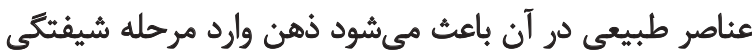

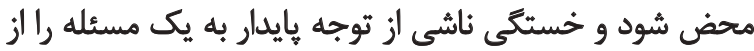

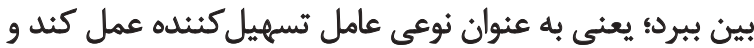
اين خود باعث بازسازي ذهن و بالا رفتن سرعت يادئيرى شودي

نظريه دلبستكى و حفظ محيط

اينورث" (19VA) و و بالبى" (19AA) اين نظريه را مطرح

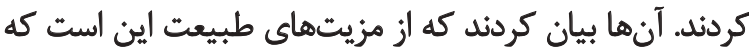

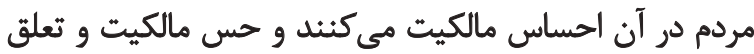

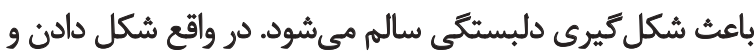

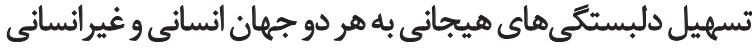

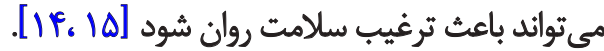
اين شيوه درمانى دربركيرنده انواع مختلفى از درمانها است

\section{Biophilia}

8. Wilson

9. Kaplan

10. Ainsworth

11. Bowlby

اختلال طيف اتيسم اختلالى است كه ملاكهاى تشخيصى

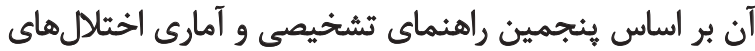

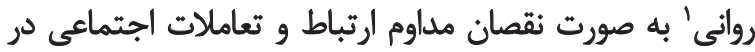

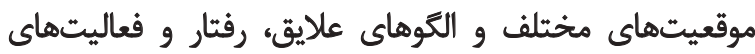

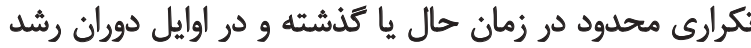

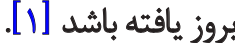

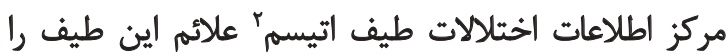

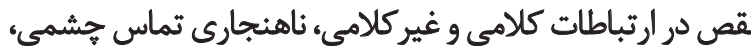

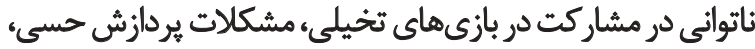

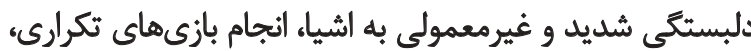

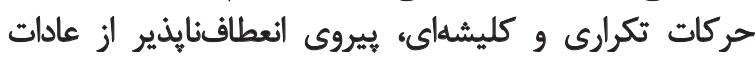

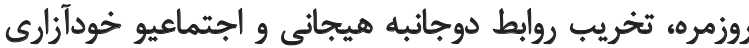

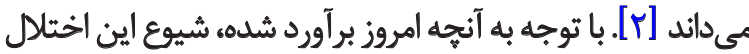

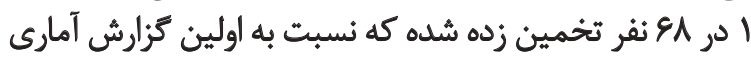

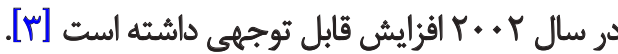

اين كودكان افرادى با عملكردهاى ذهنى متفاوت هستند. به

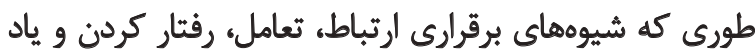

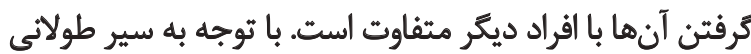

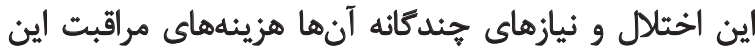

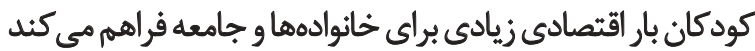
كه بخش بيشتر اين هزينهها صرف آموزش و و درمان إنهائ خاص

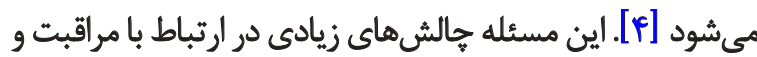

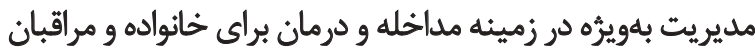

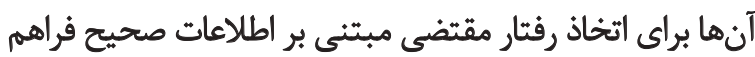

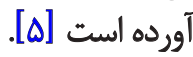

بحث درمان اين كودكان با توجه به شدت و مزمن بودن

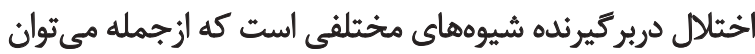

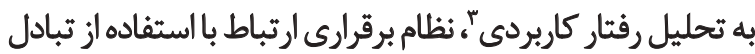

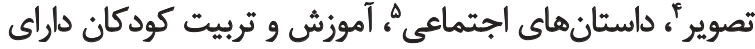

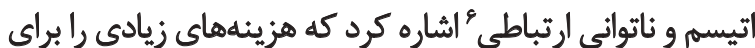

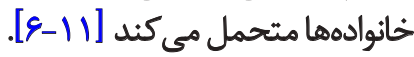

يكى ديكر از مداخلاتى كه روشى موفق و مقرونبهصرفه

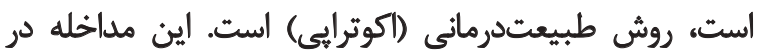

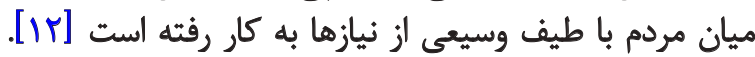

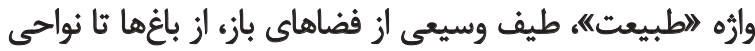

1. Diagnostic and Statistical Manual of mental disorders (DSM-5)

2. Autism Spectrum disorder Information center (SPIC)

3. Applied behavioral analysis

4. Picture Exchange Communication System (PECS)

5. Social stories

6. Treatment and Education of Autistic and related Communication Handicapped Children (TEACCH) 


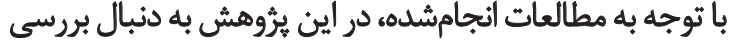

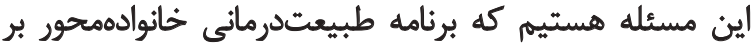

نشائكان كودكان با اختلال طيف اتيسم جه تأثيرى دارد؟

وروش بروسي

اين مطالعه به صورت نيمهآزمايشى (إيشيش آزمون و يسآزمون

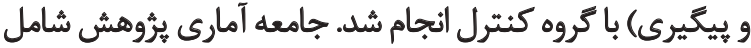

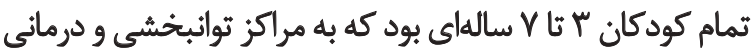

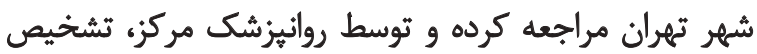

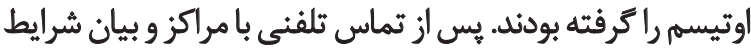

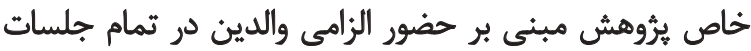

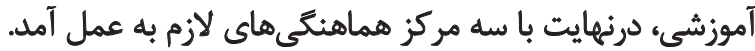

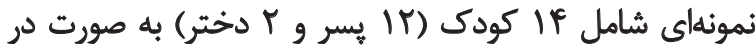

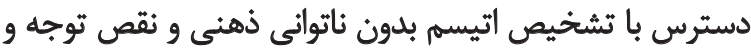

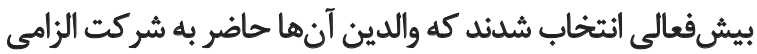

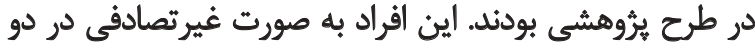

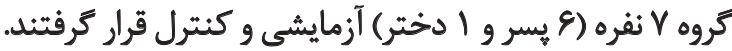
ملاكهاى خروج از مطالعه شامل وجود هركوئه اختيلال بارز

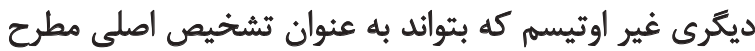

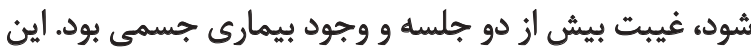

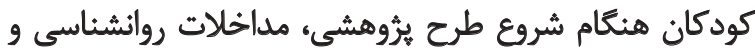

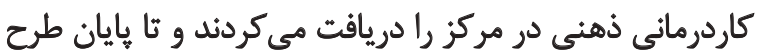

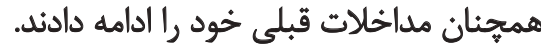

تعداد جلسات آموزشى • 1 جلسه بود كه طلى ب ماه به مدت

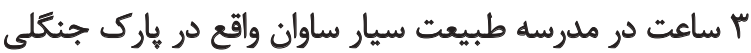

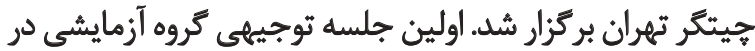

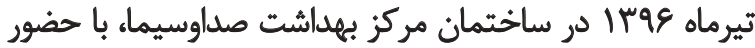

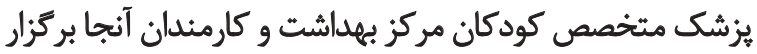

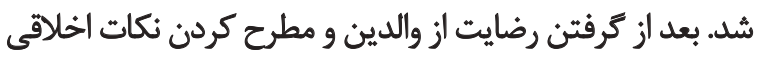

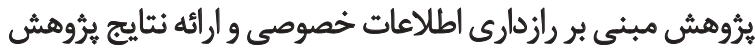

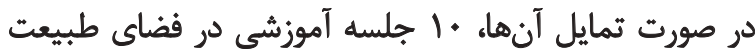

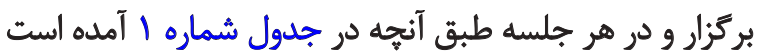
تكاليف انجام شد.

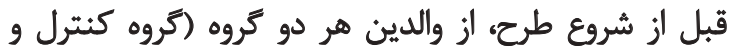

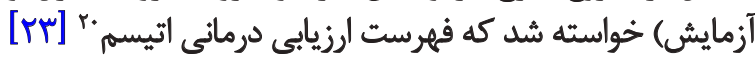

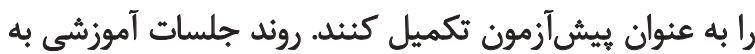

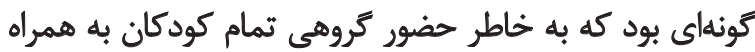

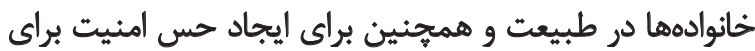

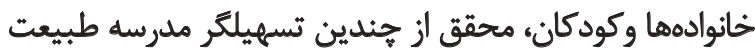

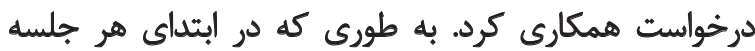
آموزشى محقق تكاليف مربوط به همان جلسه رابراى تسهيلكران بهري

20. Autism Treatment Evaluation Checklist (ATEC)

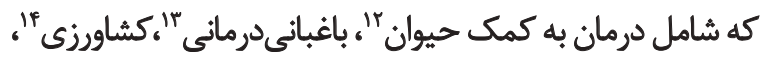

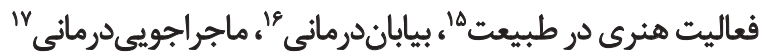

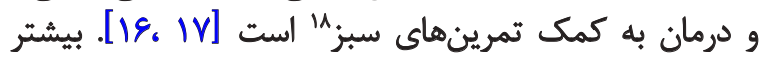

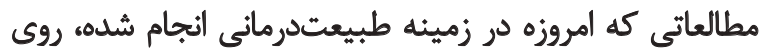

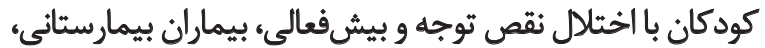

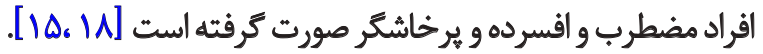

در تمام اين مطالعات ارتباط و تعامل با طبيعت اثرات مثبتى

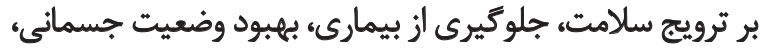

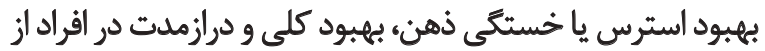

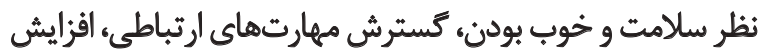

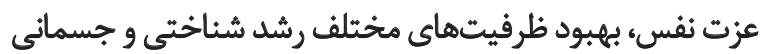

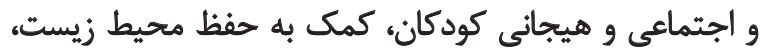

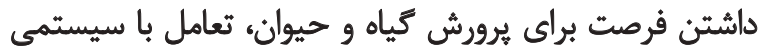

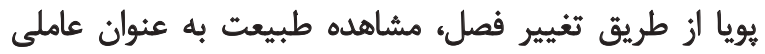

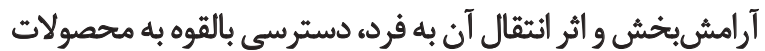

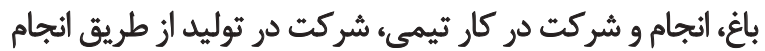

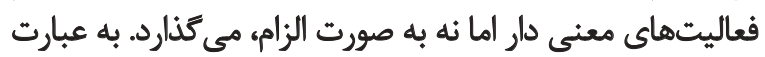
ديكر حضور در طبيعت و تعامل با عناصرى مانئ خاك، كياه،

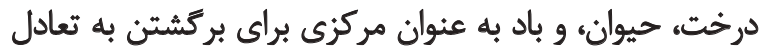

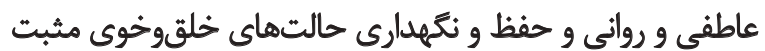

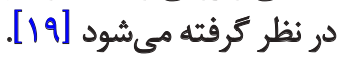

از ديكر هزيتهاي طبيعتدرماني هي توان به اين مسئله اشاره

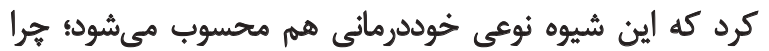

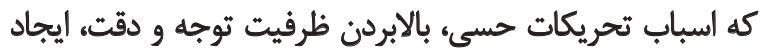
احساس امنيت، افزايش حس نشاط، كاهش استرس و خست خستيكي

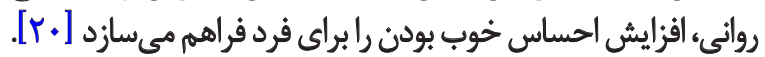
نداشتن ارتباط با طبيعت و يا عدم فعاليت كودكان در فضاى

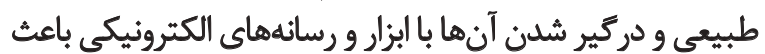

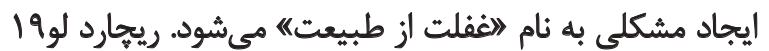

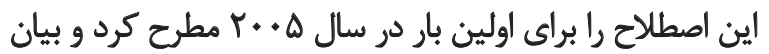

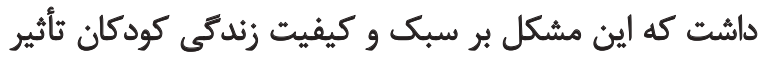

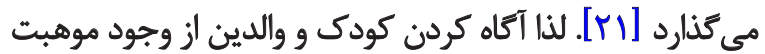
طبيعت و بيدار كرن شور و شوق حضور و بازى در طبيعت براي

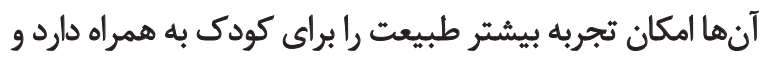

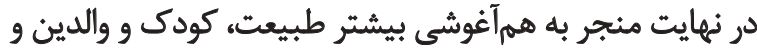

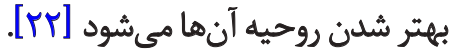

12. Animal assisted intervention

13. Horticultural therapy

14. Farming

15. Doing art in or with nature

16. Wilderness therapy

17. Adventure therapy

18. Green exercise therapy

19. Richard Louv 
دانشكاه علوم وتحقيقات تهران و مركز بهداشت صداوسيماى

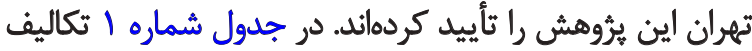
آموزشى هر جلسه آورده شده است كئ كردان.

ابزارهاى مورد استفاده در يرؤش عبارتنداز:

$$
\text { "قهرست الوزيابي دوماني اتيسي" }
$$

ريملند و ادلسون (ب991) اين فهرست را طراحى كردند.

21. Autism Treatment Evaluation Checklist (ATEC)

$$
\text { و خانوادهها توضيح مى داد و بعد آهوزش را شروع مىكرد. }
$$

هر كدام از والدين موظف بودند كه در يك سرى از تكاليف

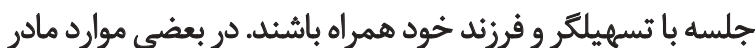

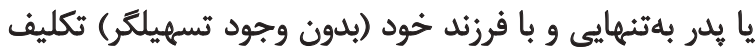

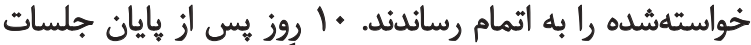

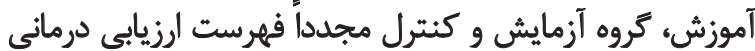

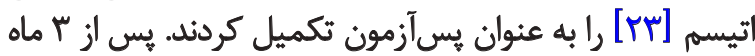
نيز تروه آزمايش آن را به عنوان مرحله بييخيرى تكميل كرد.

جدولا. تكاليف آموزشى مربوط به جلسات طبيعتدرمانى

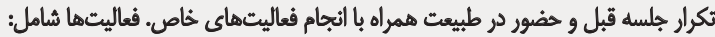

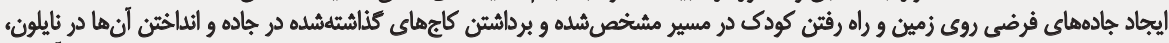

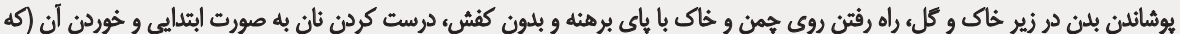

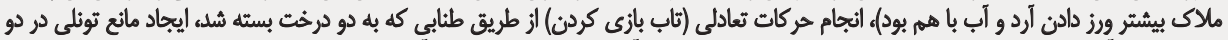

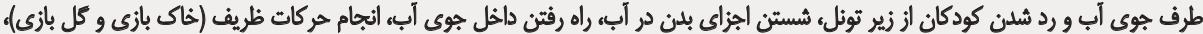

$$
\text { لمس حيون دران }
$$

تكرار جلسه قبل و حضور در طبيعت همراه با انجام فعاليتهائ خاص. فعاليتها شامل:

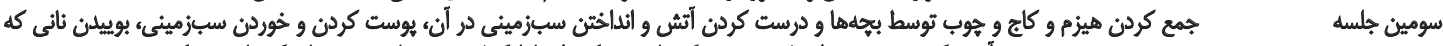

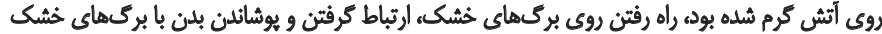

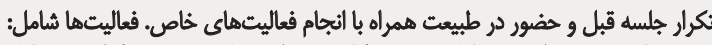

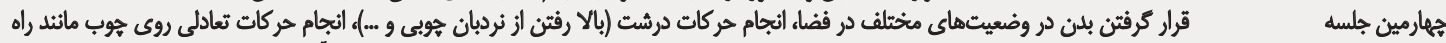

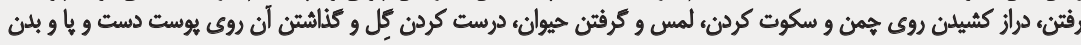

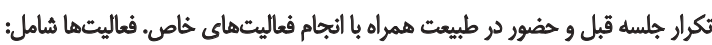

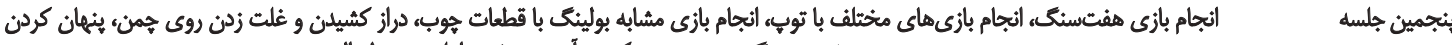

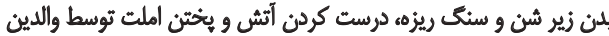

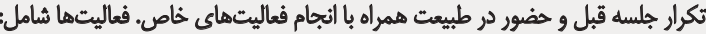

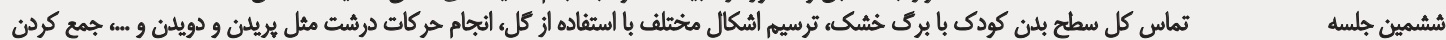

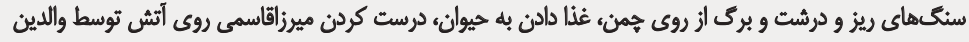

تكرار جلسه قبل و حضور در طبيعت همراه با ائجام فعاليتهاي خاص. فعاليتها شامل:

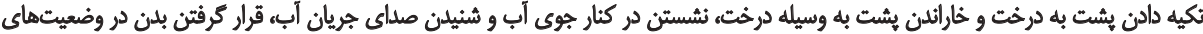

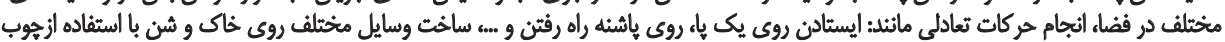

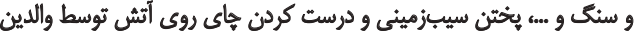

تكرار جلسه قبل و حضور در طبيعت همراه با انجام فعاليتهاي خاص. فعاليتها شامل:

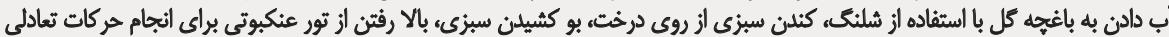
هشتمين جلسه

تكرار جلسه قبل و حضور در طبيعت همراه با انجام فعاليتهاي خاص. فعاليتها شامل:

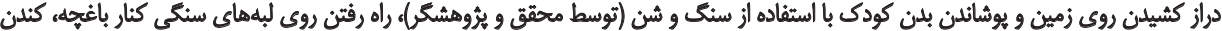

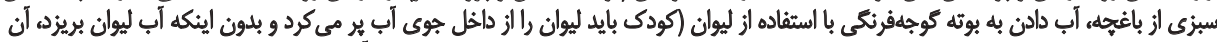
نهمين جلسه

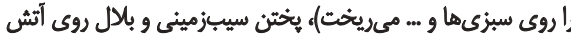

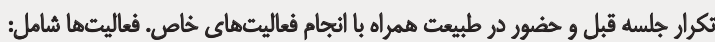

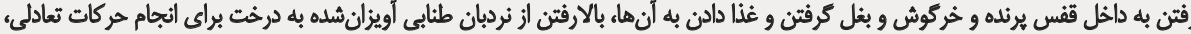

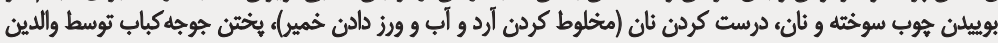


صورت آزاد و بدون هدايت مستقيم محقق صورت كرفت. مرحله

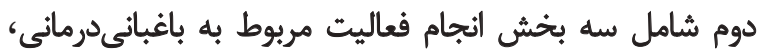

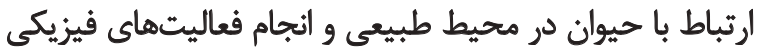

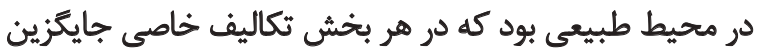

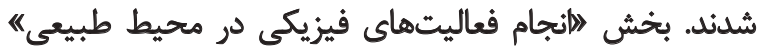

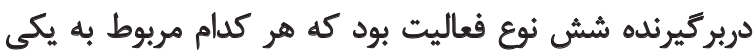

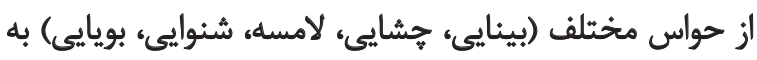

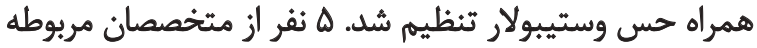

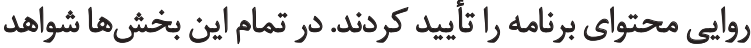

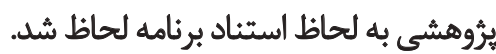

\section{يافتهما}

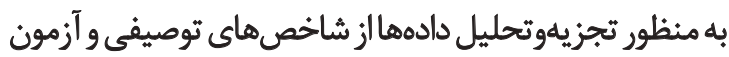

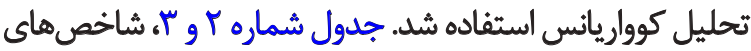

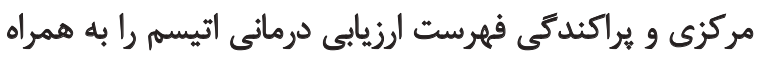

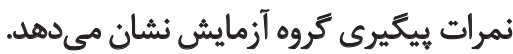

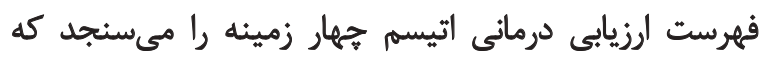

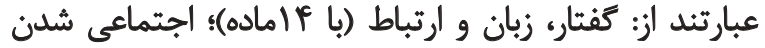

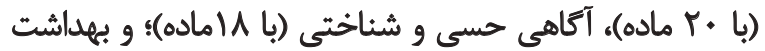

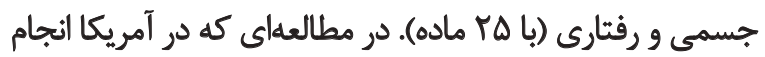

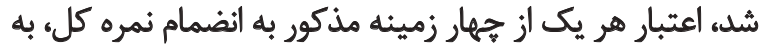

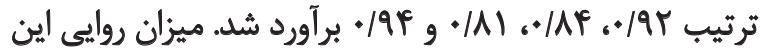

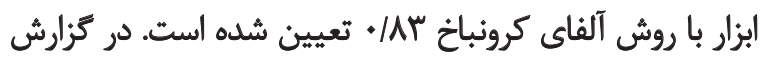

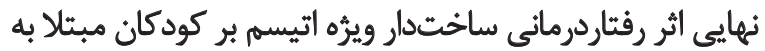

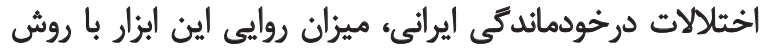

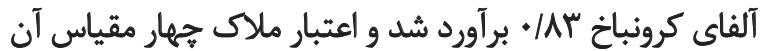

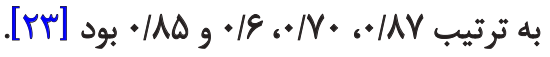
برئامه طييعت دمانى خانوادهمحور اين برنامه در دو مرحله اصلى علاقهمند شدن و دريّيرى ذهنى

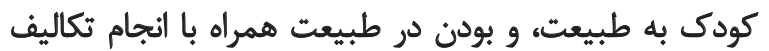

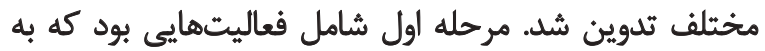
جدول T. شاخصهاى مركزى و يراكندكى فهرست ارزيابى درمانى اتيسم براى مراحل بيش آزمون و بسآزمون كروه آزمايش وكنترل

\begin{tabular}{|c|c|c|c|c|c|c|c|}
\hline بيشترين نمرات & كمترين نمرات & انعراف معيار & مياتكين & تعداد & مرحله & تروه & مقياس \\
\hline หั.. & r.\%. & $T / \pi \Delta$ & $r e / r q$ & $r$ & ييش آزمون & \multirow[b]{2}{*}{ أزمايش } & \multirow{4}{*}{ كمثتار، زيان، } \\
\hline$\varphi \%$ & $r \%$ & $P M$ & $\pi / T^{2}$ & $r$ & يسآزمون & & \\
\hline$r / .$. & $1 \% \%$ & V/rF & $\pi /$. & $r$ & ييش آزمون & \multirow{2}{*}{ كتترل } & \\
\hline$r \%$ & $1 \% \%$ & $91 . Y$ & $r y / r q$ & $r$ & يسآزمون & & \\
\hline$\Leftrightarrow \%$ & $r \%$ & $\Delta / P V$ & $T / / T$ & $r$ & ييش آزهون & \multirow[t]{2}{*}{ آزمايش } & \multirow{4}{*}{ اجتماعى } \\
\hline$\Delta V^{\circ}$. & $\varphi . \%$ & S/RT & $p q .$. & $r$ & يسأزمون & & \\
\hline$\Delta \Delta / .$. & $\pi \%$ & NFA & $r \% / r q$ & $r$ & ييش آزمون & \multirow[t]{2}{*}{ كترل } & \\
\hline$\Delta \Delta / \cdot$. & $r Q / .$. & NE. & $r T M$ & $r$ & يس آزمون & & \\
\hline$r \mathrm{~N} / .$. & r. $\%$ & $\Delta / \pi$ & $r . \%$ & $r$ & ييش آزمون & \multirow{3}{*}{ أزمايش } & \multirow{5}{*}{ و شكاهي حسىى } \\
\hline & & & & & & & \\
\hline$r \Delta / .$. & $r . \%$ & $p / 1 q$ & $r \cdot M$ & $r$ & بس آزمون & & \\
\hline$r \%$ & $\pi / .$. & $\Delta / \&$ & $\mathrm{rV} / .$. & $r$ & ييش آزهون & \multirow[b]{2}{*}{ كنترل } & \\
\hline$p / .$. & $r / .$. & $9 / 9$ & $\mathrm{rV} / .$. & $r$ & يسأزمون & & \\
\hline
\end{tabular}




\begin{tabular}{|c|c|c|c|c|c|c|c|}
\hline بيشترين نمرات & كمترين ثمرات & انتحراف معيار & مياتكين & تعداد & مرحله & كروه & مقياس \\
\hline \multirow[t]{2}{*}{ \&ql.. } & $M / *$. & $11 / 4 e$ & $\Delta 1 / P q$ & $r$ & ييش أزمون & & \\
\hline & & & & & & أزمايش & \\
\hline EVI.. & $\pi / .$. & $11 / 0$. & $\Delta \cdot / \pi$ & $\gamma$ & يسآزمون & & براشت \\
\hline \multirow[t]{2}{*}{$9 \% \%$} & $9 \% 1+\infty$ & $V / \Delta F^{\circ}$ & $\Delta 1 / 7 q$ & $r$ & ييش آزهون & & جسمى ورى \\
\hline & & & & & & كنترل & \\
\hline exp. & 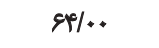 & $V / . Y$ & $\Delta \cdot / A_{0}$ & $r$ & يس آزمون & & \\
\hline \multirow[t]{2}{*}{$\mid V * \%$} & $I T N$. & $11 / \Delta$ & $1 \Delta T / Y$ & $v$ & ييشآزمون & & \\
\hline & & & & & & آزمايش & \\
\hline $194 \%$ & $\mid \Delta F /$ & $11 / \Delta$ & $\mid V T / r$ & $r$ & يس آزممون & & \\
\hline \multirow[t]{2}{*}{ มข. } & We & $10 / 94$ & $\mid Q 1 / 9$ & $r$ & ييش آزمون & & \\
\hline & & & & & & كتترل & \\
\hline $\mid A V /$. & $I V / V$ & $r i /$. & $|\Delta| / \mid$ & $\checkmark$ & يس آرمون & & \\
\hline
\end{tabular}

توانبخننى

r مشاهده مىشود نمرات بيخيرى گروه آزمايش لحاظ شدهاند.

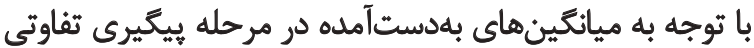

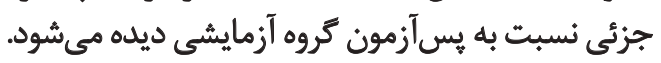

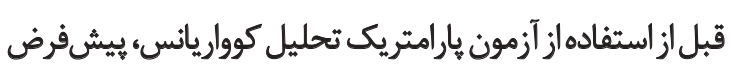

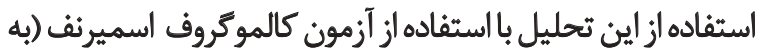

همانطوركه در جدول شماره r مشاهده مي آشود، ميانگين

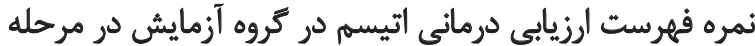

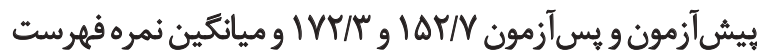

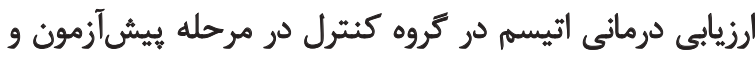

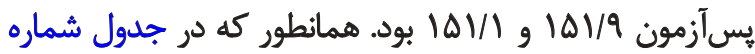

جدول ץ. ميانكين و انحرافمعيار مقياسها در بس آزمون كروه آزمايش و آزمون بيكيرى

\begin{tabular}{|c|c|c|c|}
\hline انحراف استاندارد & ميانكين & مرحلد & شاخص \\
\hline$F / 1$ & $\pi / \pi q$ & يس أزمون & \multirow{2}{*}{ كفتار، زيان، ارتباطات } \\
\hline$P / \Delta V$ & $r \Delta / w$ & ييكيرى & \\
\hline $8 / \pi$ & $p q / .$. & بس أزمون & \multirow{2}{*}{ اجتماعى شلن } \\
\hline$v / 1$. & $\Delta V / T T$ & بيكيرى & \\
\hline$r / 19$ & $r \cdot M$ & يس أزمون & \multirow{2}{*}{ كاهى حسى و شناختى } \\
\hline r/ar & $r . / 9$. & بيكيرى & \\
\hline $11 / \Delta$. & $\Delta \cdot / \pi$ & يس آزمون & \multirow{2}{*}{ بهالشت } \\
\hline W/9 & $\Delta \cdot(\Delta)$ & بيكيرى & \\
\hline $11 / \Delta$ & $\mid v / r$ & يس آزمون & \multirow{2}{*}{ كل } \\
\hline $\mid r / . r$ & $1 n / 9$. & بيكيرى & \\
\hline
\end{tabular}


جدول f. أثتايج تحليل كوواريائس در بررسى اثربخشى برئامه طبيعتدرمانى بر نشانكان طيف اتيسم.

\begin{tabular}{|c|c|c|c|c|c|}
\hline اتا & سطح معنادارى & $F$ & درجه آزادى & مجموع مجذورات & منيع شاخص تغييرات \\
\hline \multirow[t]{3}{*}{.190} & & $\mathrm{rI/q}$. & 1 & IFE.1.r & أزمايش \\
\hline & & & 11 & $n T / r$. & خطا \\
\hline & .10 .1 & & r & PTYNA & كل \\
\hline
\end{tabular}

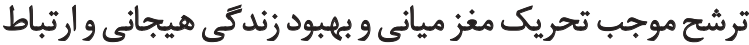

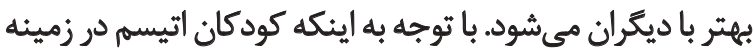

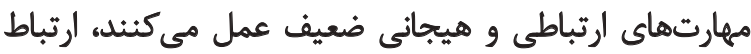

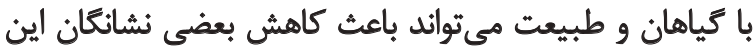
كودكان شود [ry]

تبيين ديكرى كه ميتوان ارائه كرد نظرات ويلسون است.

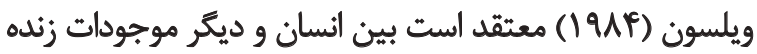

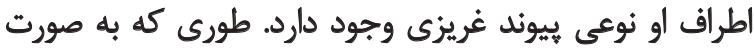

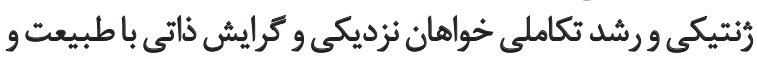

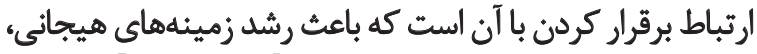

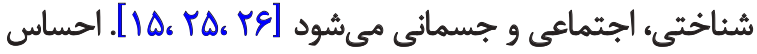

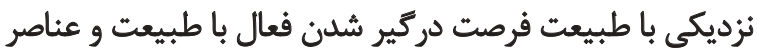

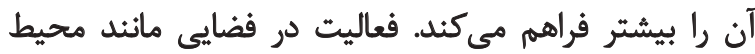

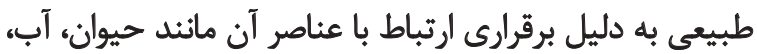

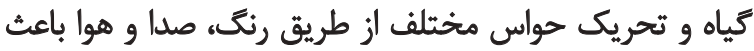

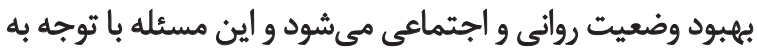

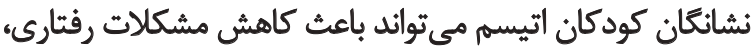

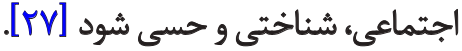

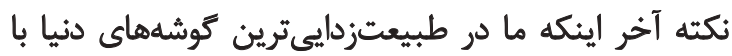

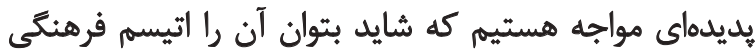

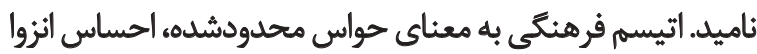

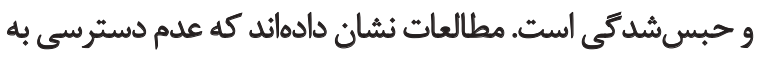

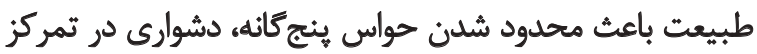

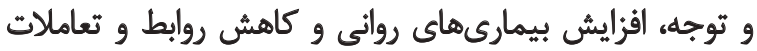

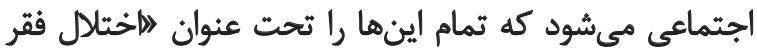

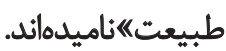

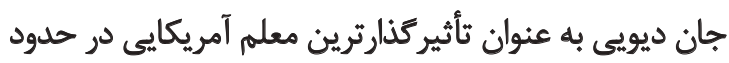

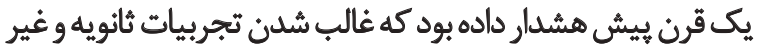

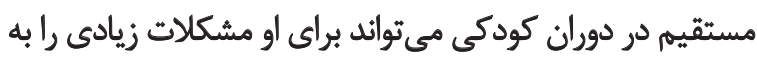

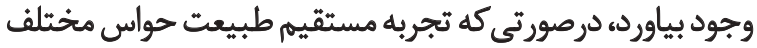

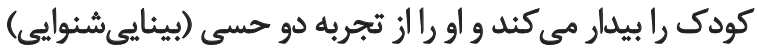

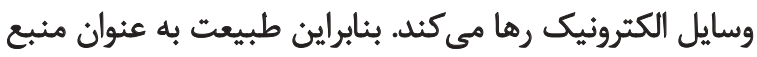

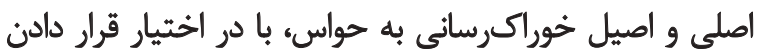

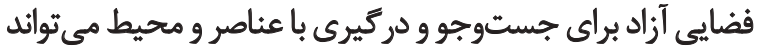

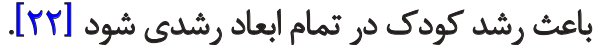

منظور بررسى طبيعى بودن توزيع) و آزمون لون (به منظور بررسى ئري

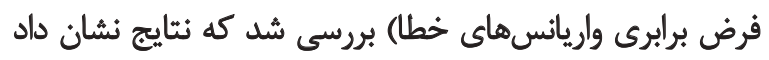

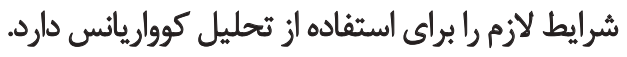

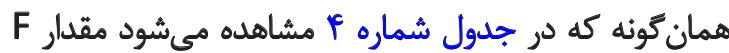

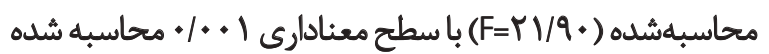

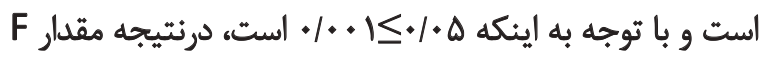

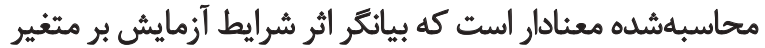

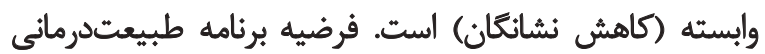

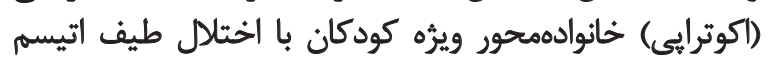

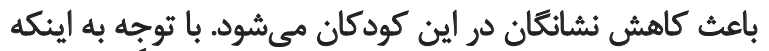

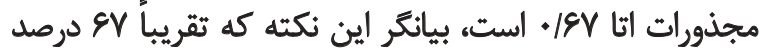

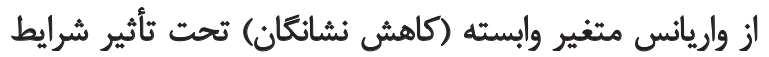
آزمايش قرار دارد.

ث્!

هدف اصلى يروهش حاضر اثربخشى طبيعتدرمانى (اكوترايى)

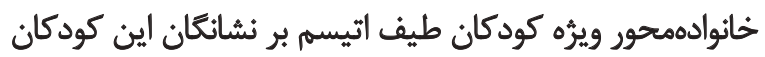

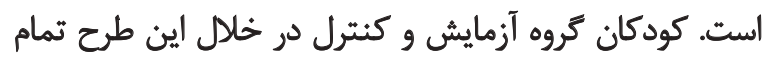

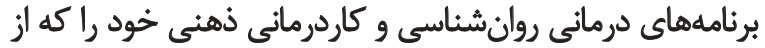

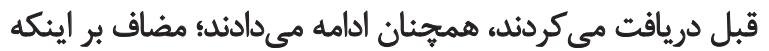

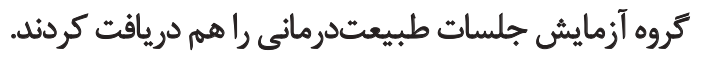

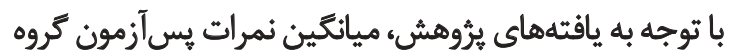

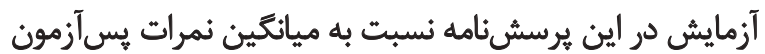

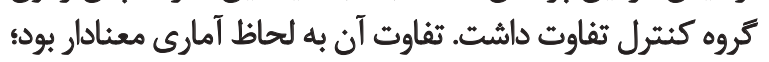

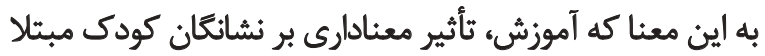

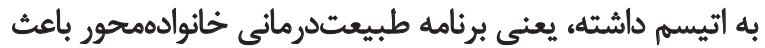
كاهش نشانكان شده است.

در تبيين يافتههاي اين يُروهش بايد به تئورى سهكانه مغز

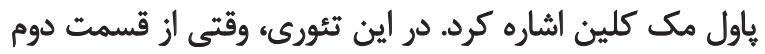

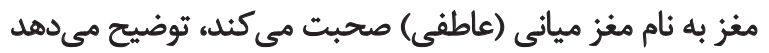

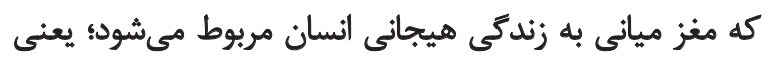

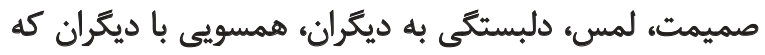

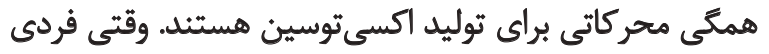

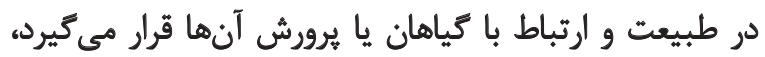

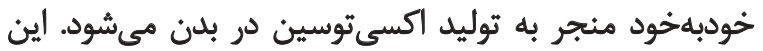




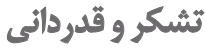

اين مقاله از رساله دكتراى خانم مريم رامشينى از زائروه

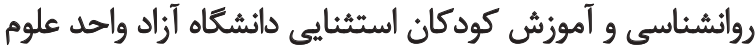

وتحقيقات تهران گرفته شده است. مقاله حامي مالى ندارد.

بدينوسيله از دكتر حميد حسينى (رياست محترم مركز

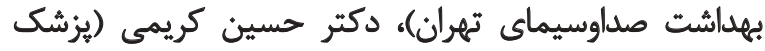

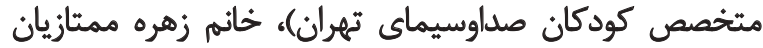

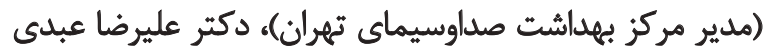

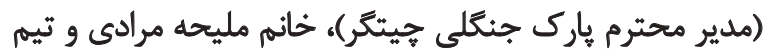

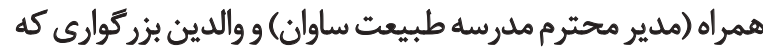

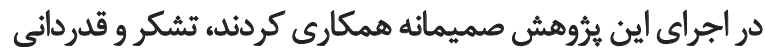

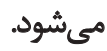

يافتههاى اين يُروهش با نتايج مطالعات تازيكى و همكاران

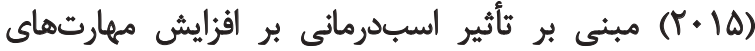

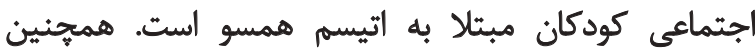

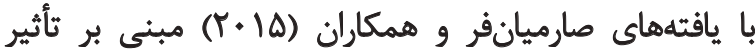

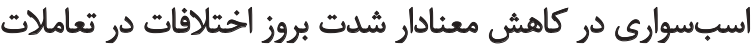

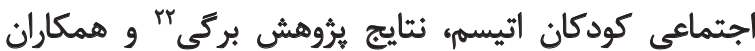

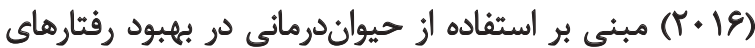

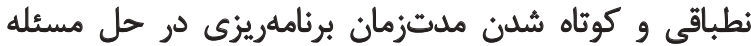

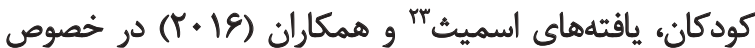

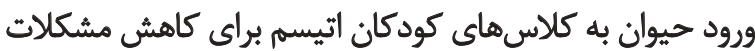

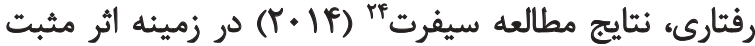

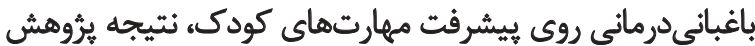

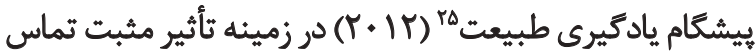

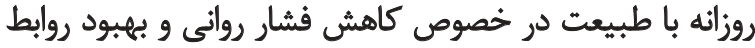

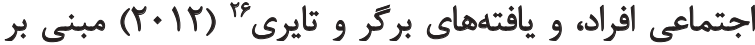

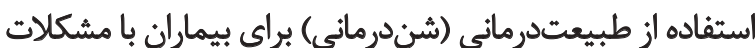

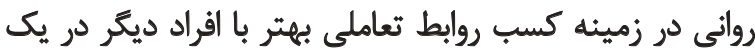

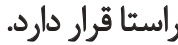

\section{نتيجهيَيرى}

بر اساس يافتهاى يُروهش حاضر مىتوان نتيجه كرفت كه

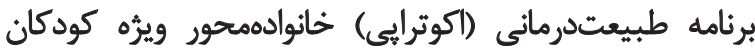

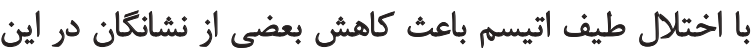

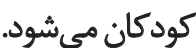

از محدوديتهاى ثيروهش حاضر مي توان به فضاى مدرسه

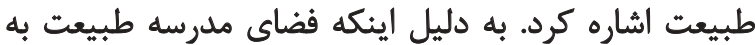
طور كلى براى كودكان عادى تعبيه شده است و أز از نظر ابزار

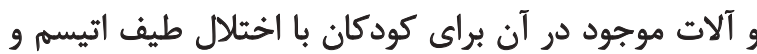

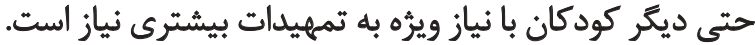

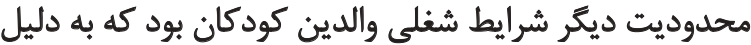

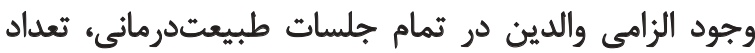

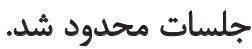

با توجه به اينكه اين برنامه براى كودكان و به دليل خانوادهمحور

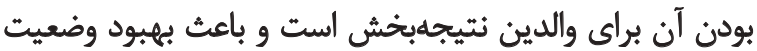

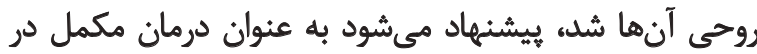

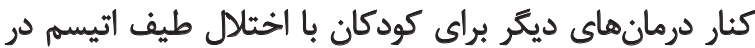

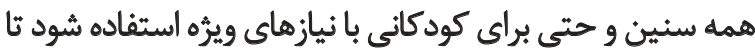

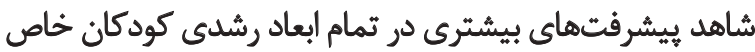

$$
\text { و سلامت روان بيشتر خانوادههاى آنها باشئمانيم. }
$$

\section{Borgi}

23. Smith

24. Sifret

25. Natural learning initiative

26. Berger and Tiry 


\section{References}

[1] American Psychiatric Association. Diagnostic and statistical manual of mental disorders [F Rezaee, et al., Persian trans.]. Tehran: Arjmand Pub; 2013.

[2] Murphy R. SPICe briefing: Autism spectrum disorder. Edinburgh: Scottish Parliament Information Centre; 2017.

[3] Russell S, McCloskey CR. Parent perceptions of care received by children with an autism spectrum disorder. Journal of Pediatric Nursing. 2016; 31(1):21-31. [DOI:10.1016/j.pedn.2015.11.002]

[4] Centers for Disease Control and Prevention (CDC). A snapshot of autism spectrum disorder among 8-year-old children in Multiple Communities across the United States. Atlanta: United States Department of Health and Human Services; 2014.

[5] Hsiao YJ. Pathways to mental health-related quality of life for parents of children with autism spectrum disorder: roles of parental stress, children's performance, medical support, and neighbor support. Research in Autism Spectrum Disorders. 2016; 23:12230. [DOI:10.1016/j.rasd.2015.10.008]

[6] Golzari F, Hemati Alamdarloo G, Moradi S. The effect of a social stories intervention on the social skills of male students with autism spectrum disorder. SAGE Open. 2015; 5(4):215824401562159. [DOI:10.1177/2158244015621599]

[7] Morgenthal AH. Child-centered play therapy for children with autism: A case study (PhD dissertation). Culver City, California: Antioch University; 2015.

[8] Welterlin A, Turner Brown LM, Harris S, Mesibov G, Delmolino L. The home teacching program for toddlers with autism. Journal of Autism and Developmental Disorders. 2011; 42(9):1827-35. [DOI:10.1007/s10803-011-1419-2]

[9] Politte LC, Howe Y, Nowinski L, Palumbo M, McDougle CJ. Evidence-based treatments for autism spectrum disorder. Current Treatment Options in Psychiatry. 2015; 2(1):38-56. [DOI:10.1007/s40501-015-0031-z]

[10] Leaf JB, Leaf R, McEachin J, Taubman M, Ala'i-Rosales S, Ross $\mathrm{RK}$, et al. Applied behavior analysis is a science and, therefore, progressive. Journal of Autism and Developmental Disorders. 2015; 46(2):720-31. [DOI:10.1007/s10803-015-2591-6]

[11] Battaglia D, McDonald ME. Effects of the Picture Exchange Communication System (PECS) on maladaptive behavior in children with Autism Spectrum Disorders (ASD): A review of the literature. Journal of the American Academy of Special Education Professionals. 2015; 8:20.

[12] Seifert AR. Cultivating new lives: Anethnographic pilot study of Eco-therapy provision for people with Alcohol-related problems in Northern Ireland. Anthropology in Action. 2014; 21(1):4-12. [DOI:10.3167/aia.2014.210103]

[13] Corazon SS, Stigsdotter UK, Jensen AG, Nilsson K. Development of the nature-based therapy concept for patients with stressrelated illness at the Danish healing forest garden Nacadia. Journal of Therapeutic Horticulture. 2010; 20:33-51.

[14] Plambech T, Konijnendijk van den Bosch CC. The impact of nature on creativity: A study among Danish creative profession- als. Urban Forestry \& Urban Greening. 2015; 14(2):255-63. [DOI:10.1016/j.ufug.2015.02.006]

[15] Adams M, Jordan M, Wren J, Wright J. The grow project: A report on the well-being benefits of nature connection for people with experience of mental distress. Brighton, UK: University of Brighton, Community and University Partnership Project. 2014.

[16] Sempik J. Green care and mental health: Gardening and farming as health and social care. Mental Health and Social Inclusion. 2010; 14(3):15-22. [DOI:10.5042/mhsi.2010.0440]

[17] Mind for Better Mental Health. Making sense of ecotherapy [Internet]. 2015. Availble from: https://mhaw.nz/assets/Overseaslit-PDFs/making-sense-of-ecotherapy-MIND-2013.pdf

[18] Mustapa ND, Maliki NZ, Hamzah A. Repositioning children's developmental needs in space planning: A review of connection to nature. Procedia-Social and Behavioral Sciences. 2015; 170:330-9. [DOI:10.1016/j.sbspro.2015.01.043]

[19] Kahn PH, Kellert SR. Children and nature: Psychological, sociocultural, and evolutionary investigations. Cambridge: MIT Press; 2002 .

[20] Brown DK, Barton JL, Pretty J, Gladwell VF. Walks4Work: Assessing the role of the natural environment in a workplace physical activity intervention. Scandinavian Journal of Work, Environment \& Health. 2014; 40(4):390-9. [DOI:10.5271/sjweh.3421]

[21] McCurdy LE, Winterbottom KE, Mehta SS, Roberts JR. Using nature and outdoor activity to improve children's health. Current Problems in Pediatric And Adolescent Health Care. 2010; 40(5):102-17. [DOI:10.1016/j.cppeds.2010.02.003]

[22] Louv R. Last child in the woods: Saving our childran from nature-deficit disorder [A Hosseinian, F Azad, Persian trans.]. Tehran: Talangor Pub; 2016.

[23] Pouretemad HR, Khooshabi K. [The effect of autism therapeutic behavioral therapy-Lovaas on children with autistic disorders (Persian)]. Tehran: Shahid Beheshti University of Medical Sciences; 2004.

[24] Haller RL, Kramer CL. Horticultural therapy methods: Connecting people and plants in health care, human services, and therapeutic programs. Abingdon: CRC Press; 2017.

[25] Bagot KL, Allen FC, Toukhsati S. Perceived restorativeness of children's school playground environments: Nature, playground features and play period experiences. Journal of environmental psychology. 2015; 41:1-9. [DOI:10.1016/j.jenvp.2014.11.005]

[26] Rogerson M, Barton J. Effects of the visual exercise environments on cognitive directed attention, energy expenditure and perceived exertion. International Journal of Environmental Research and Public Health. 2015; 12(7):7321-36. [DOI:10.3390/ ijerph120707321] [PMID] [PMCID]

[27] Rat W. Nature and the child (Practical guideline for nature schools) [AH Vahabzade, Persian trans.]. Mashhad: Sahra Pub; 2016. 